\title{
Comparative Quantum Chemistry Study on the Unimolecular Decomposition Channels of Pyrazole and Imidazole Energetic Materials
}

\author{
Shuangfei Zhu, $\dagger$ Wei Yang, $\neq$ Qiang Gan, *†, and Changgen Feng $†$
}

$\uparrow$ State Key Laboratory of Explosion Science and Technology, Beijing Institute of

Technology, Beijing 100081, China

$¥$ School of Materials Science \& Engineering, Beijing Institute of Technology, Beijing 100081, China

*Email: ganqiang@bit.edu.cn 
Table S1. Detailed data of LBO and BDE for Figure 3.

\begin{tabular}{|c|c|c|c|}
\hline bond & molecule & LBO & BDE $(\mathrm{kcal} / \mathrm{mol})$ \\
\hline \multirow{4}{*}{$\mathrm{C}-\mathrm{C}$} & $\mathrm{CH} 3-\mathrm{CH} 3$ & 1.00478 & 90.2 \\
\cline { 2 - 4 } & $\mathrm{CH} 2=\mathrm{CH} 2$ & 1.96546 & 174.1 \\
\cline { 2 - 4 } & $\mathrm{CH} \equiv \mathrm{CH}$ & 2.81885 & 228 \\
\hline \multirow{4}{*}{$\mathrm{C}-\mathrm{N}$} & $\mathrm{CH} 3-\mathrm{NH} 2$ & 0.74174 & 84.1 \\
\cline { 2 - 4 } & $\mathrm{CH} 2=\mathrm{N} \cdot$ & 2.08727 & 150 \\
\cline { 2 - 4 } & $\mathrm{CH} \equiv \mathrm{N}$ & 2.86793 & 223.4 \\
\hline \multirow{4}{*}{$\mathrm{N}-\mathrm{N}$} & $\mathrm{NH} 2-\mathrm{NH} 2$ & 0.48183 & 65.5 \\
\cline { 2 - 4 } & $\mathrm{NH}=\mathrm{NH}$ & 1.71221 & 119.7 \\
\cline { 2 - 4 } & $\mathrm{N} \equiv \mathrm{N}$ & 3.37798 & 225.81 \\
\hline
\end{tabular}




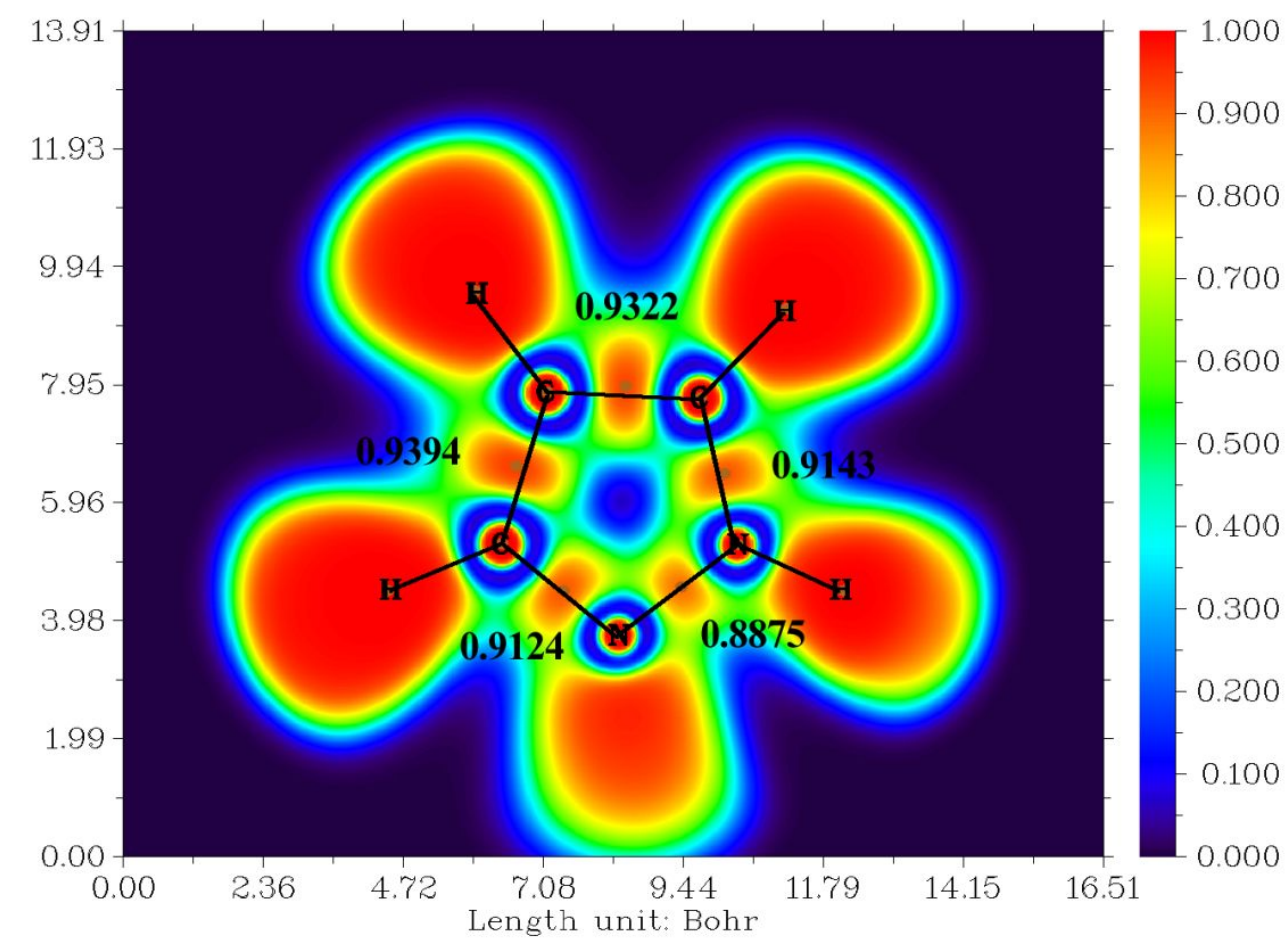

Figure S1. Electron localization function (ELF) analysis of PA, the number represents the maximum ELF value of the main chemical bond. 


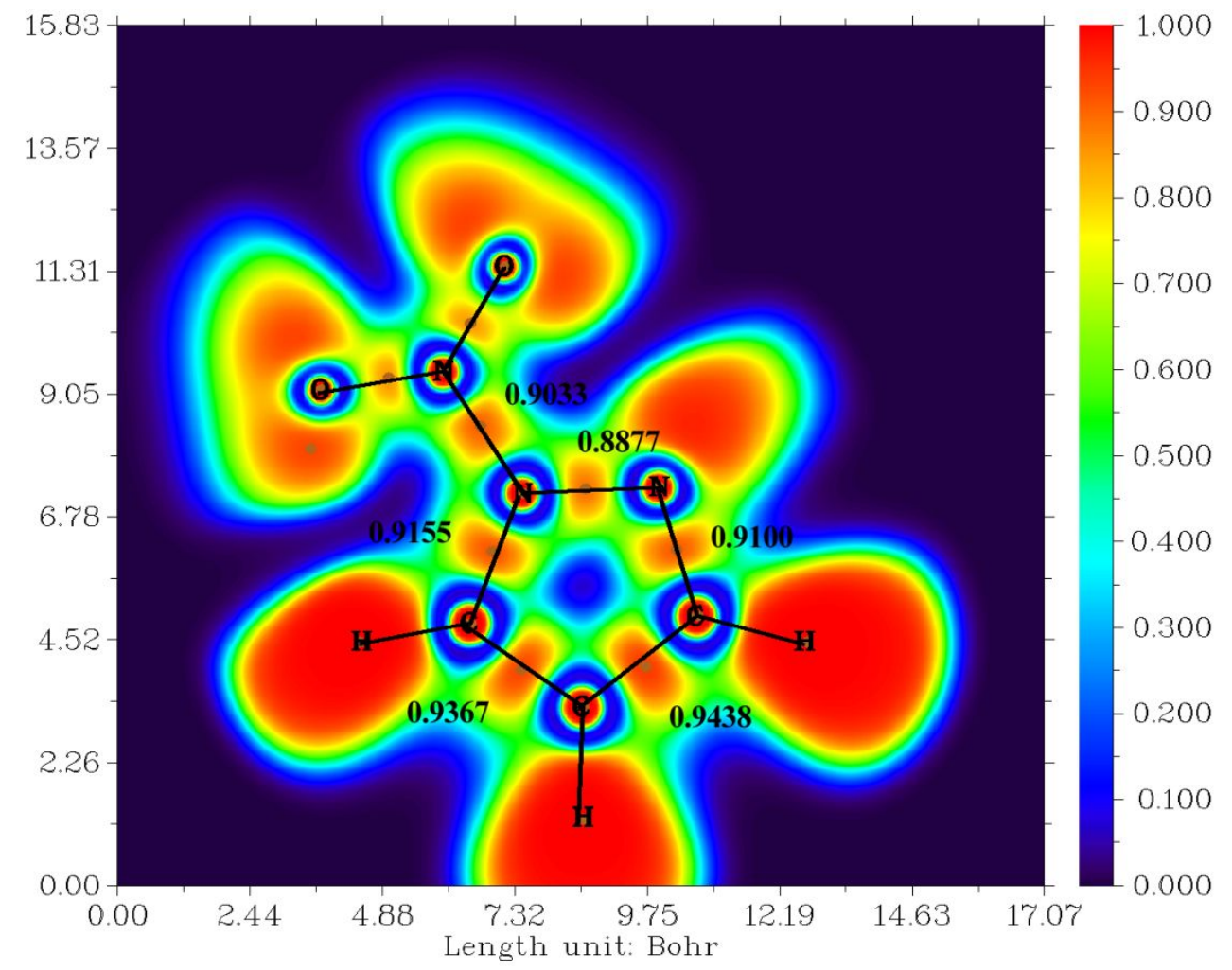

Figure S2. Electron localization function (ELF) analysis of 1NPA, the number represents the maximum ELF value of the main chemical bond. 


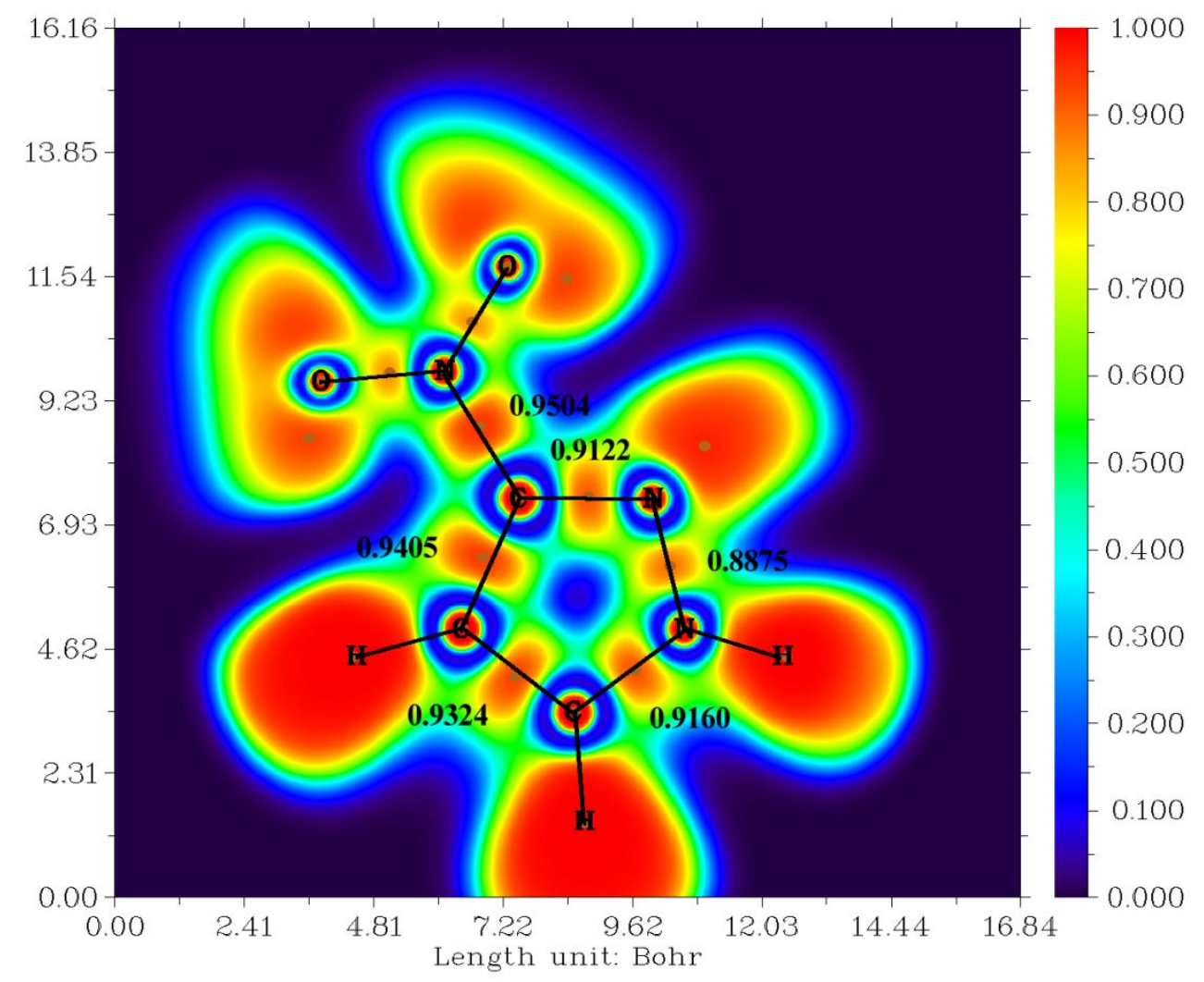

Figure S3. Electron localization function (ELF) analysis of 3NPA, the number represents the maximum ELF value of the main chemical bond. 


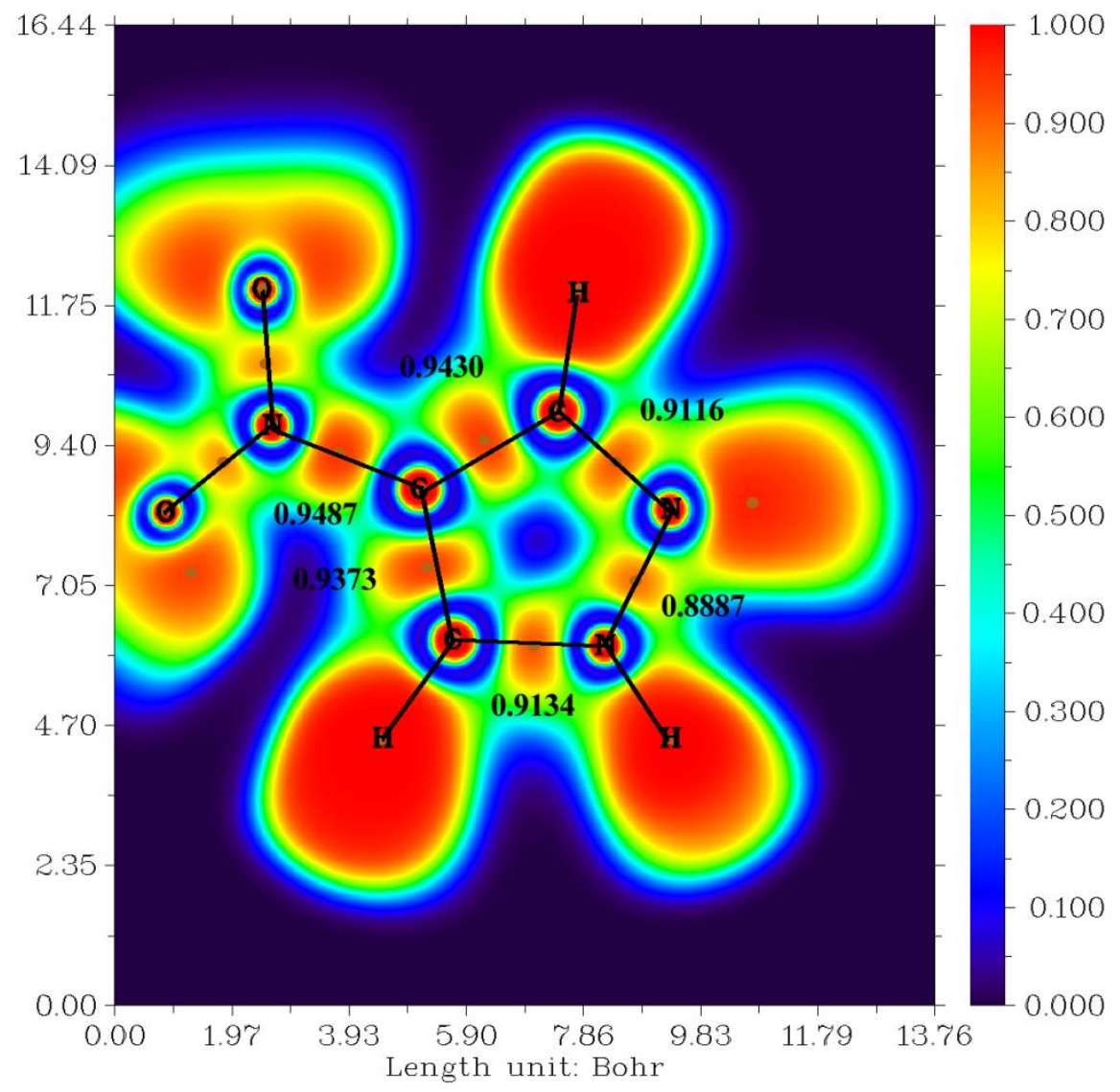

Figure S4. Electron localization function (ELF) analysis of 4NPA, the number represents the maximum ELF value of the main chemical bond. 


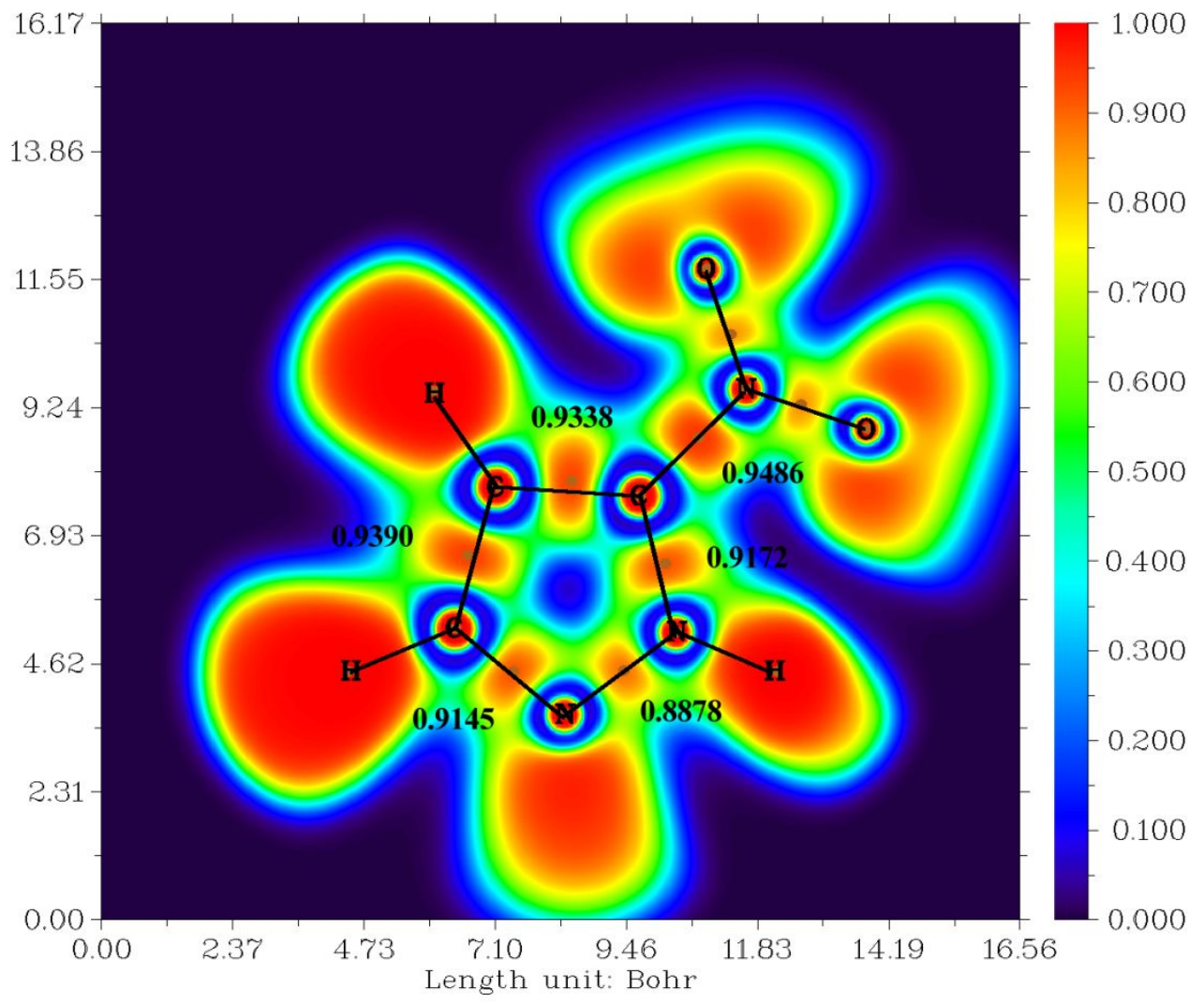

Figure S5. Electron localization function (ELF) analysis of 5NPA, the number represents the maximum ELF value of the main chemical bond. 


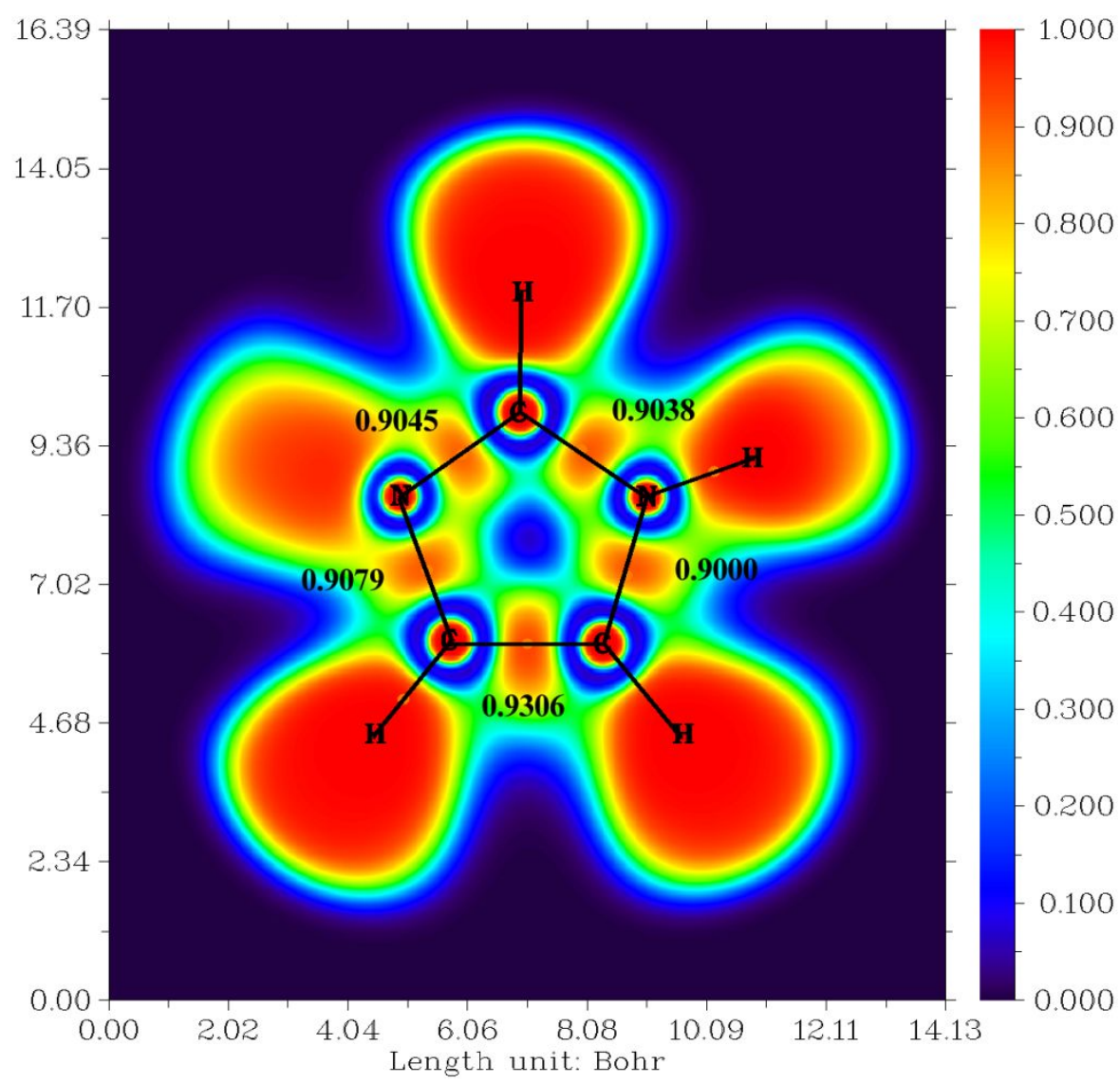

Figure S6. Electron localization function (ELF) analysis of IA, the number represents the maximum ELF value of the main chemical bond. 


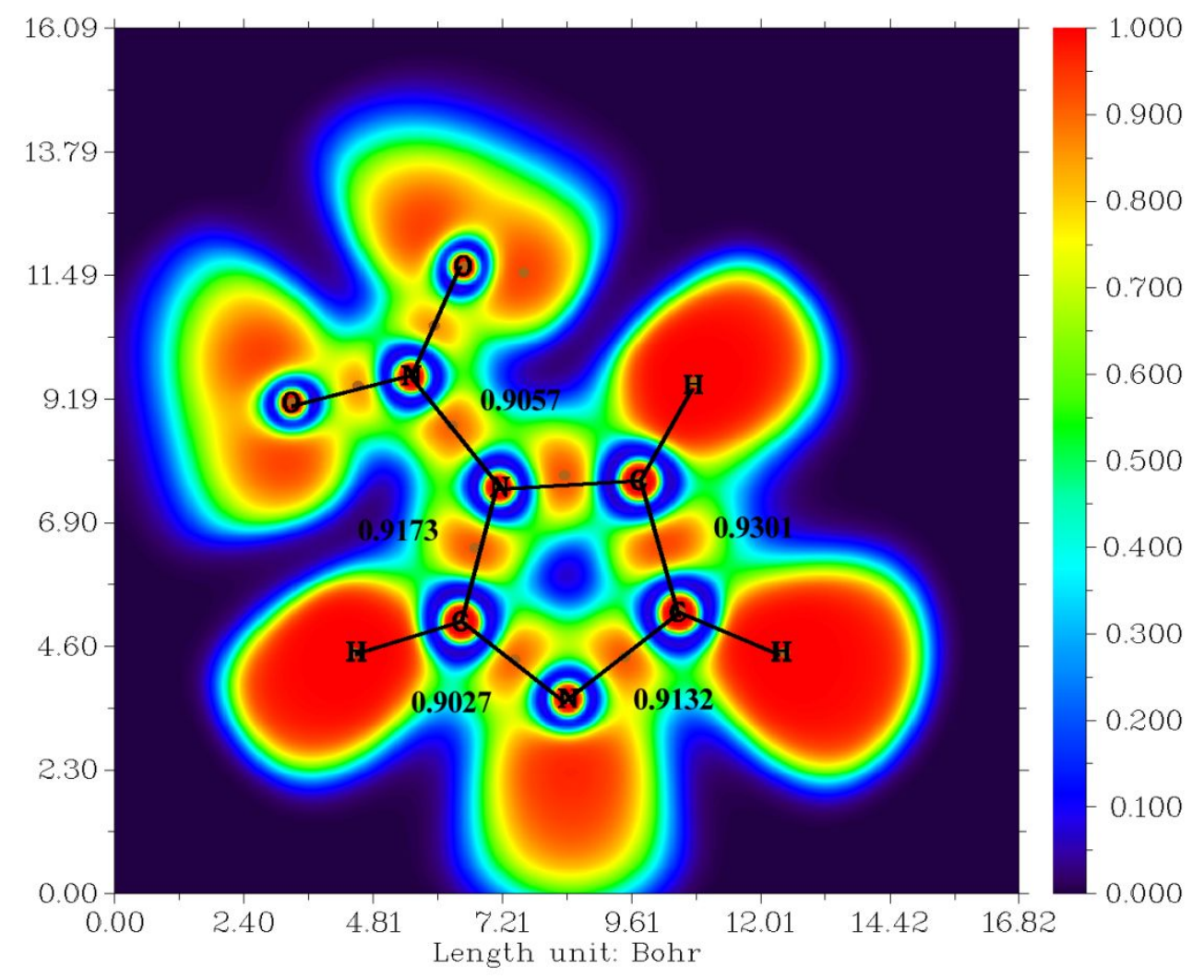

Figure S7. Electron localization function (ELF) analysis of 1NIA, the number represents the maximum ELF value of the main chemical bond. 


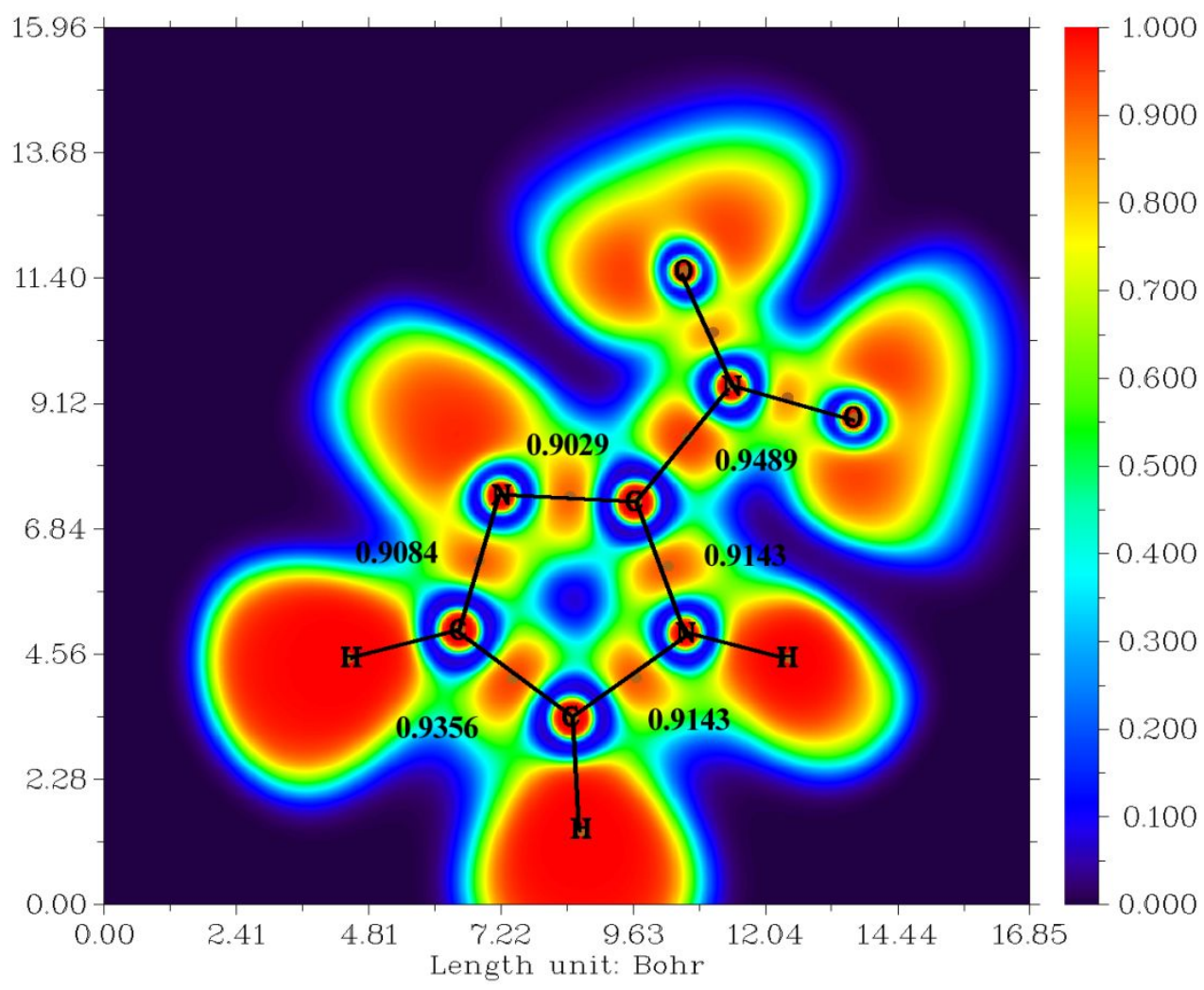

Figure S8. Electron localization function (ELF) analysis of 2NIA, the number represents the maximum ELF value of the main chemical bond. 


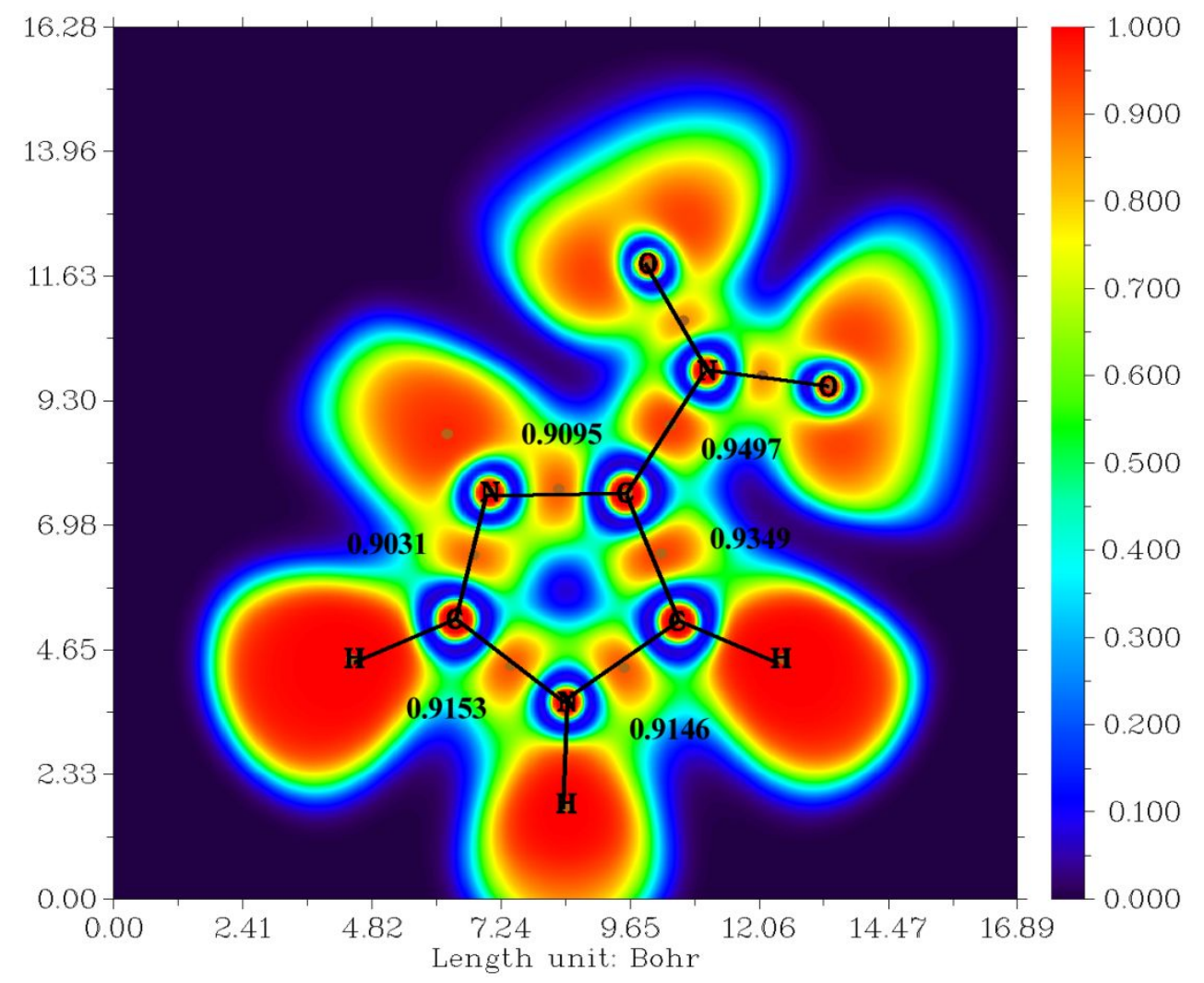

Figure S9. Electron localization function (ELF) analysis of 4NIA, the number represents the maximum ELF value of the main chemical bond. 


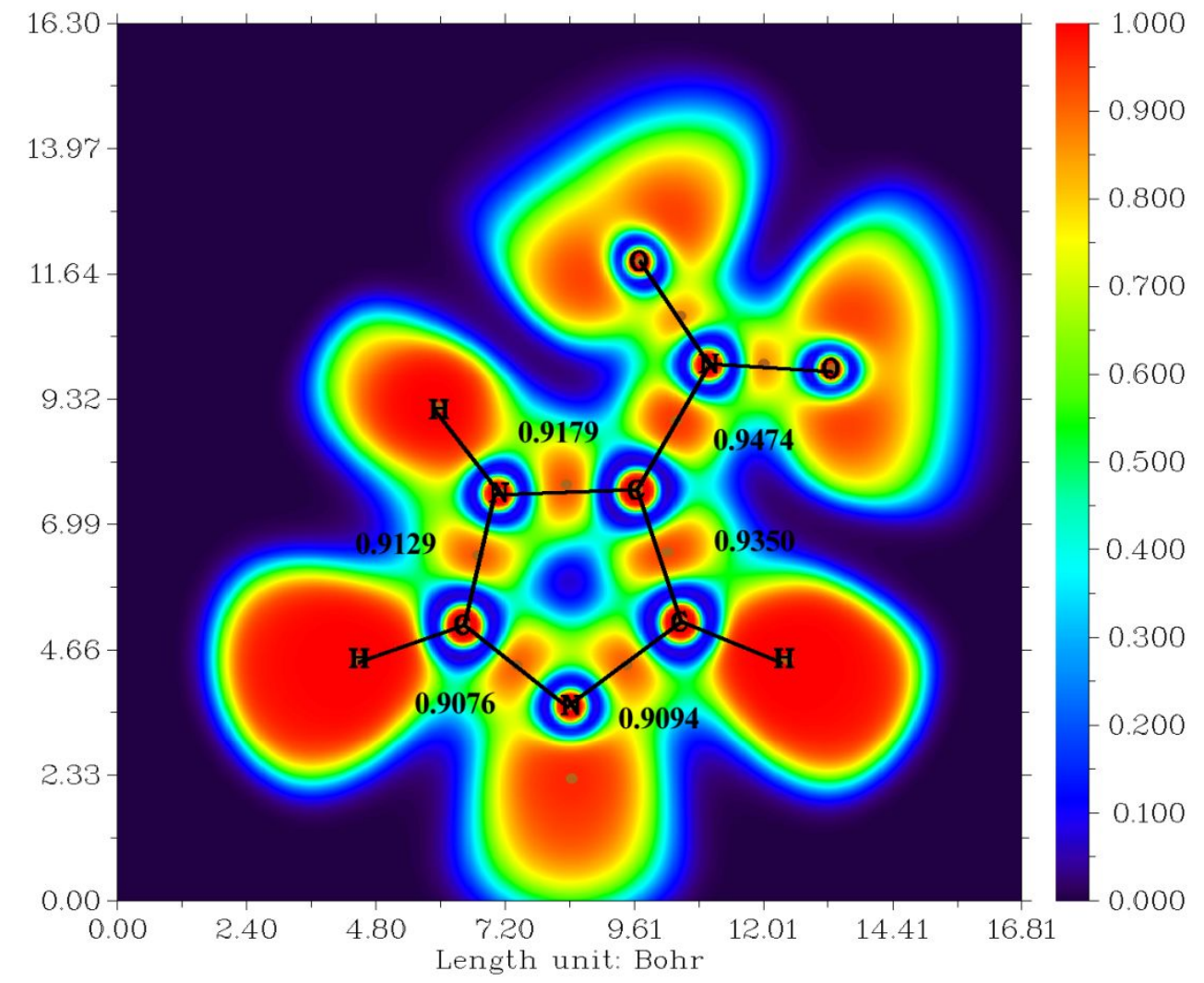

Figure S10. Electron localization function (ELF) analysis of 5NIA, the number represents the ELF maximum of the main chemical bond. 

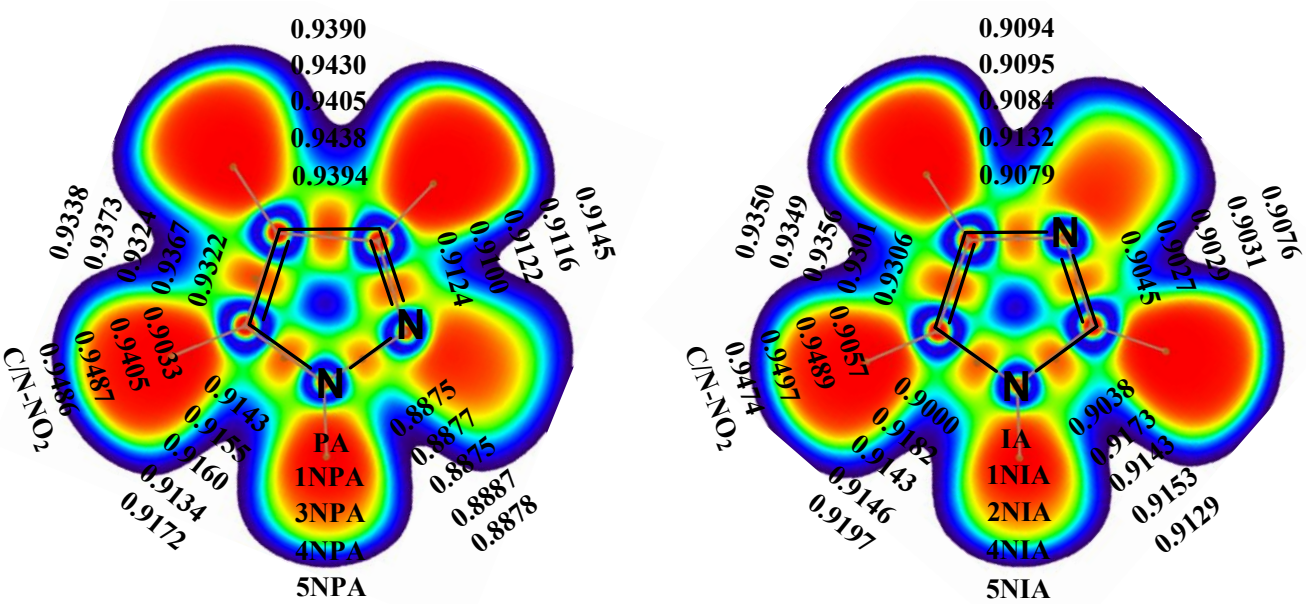

Figure S11. Electron localization function (ELF) analysis of PA, IA and their nitro derivatives Electron localization function (ELF) is an important real-space function for studying the electronic structure of chemical systems. ELF is within the range of [0,1]. A large ELF value means that electrons are greatly localized, indicating that there is a covalent bond, a lone pair or inner shells of the atom involved. Figure S1-S10 shows the ELF maps of PA, IA and their nitro derivatives. Figure S11 shows the ELF color filled maps and the ELF maximum upon the bond. 
In the case of barrierless radical decomposition reactions, the accurate computation of the rate constant (e.g., using variational transition state theory) is a rather demanding procedure. The scan of the PES along the reaction coordinate requires the use of demanding multi-reference quantum chemical procedures. Moreover, the transition state localized in such a way has a number of hindered rotations, which cannot be properly accounted using the simple conventional rigid rotorharmonic oscillator (RRHO) approximation. Thus, in our case we simply estimated the higher limit of the preexponential factor as $\log \mathrm{A}=18.0$ using the vast literature data on the radical decomposition of aliphatic and aromatic nitrocompounds (refs 43 and 44).
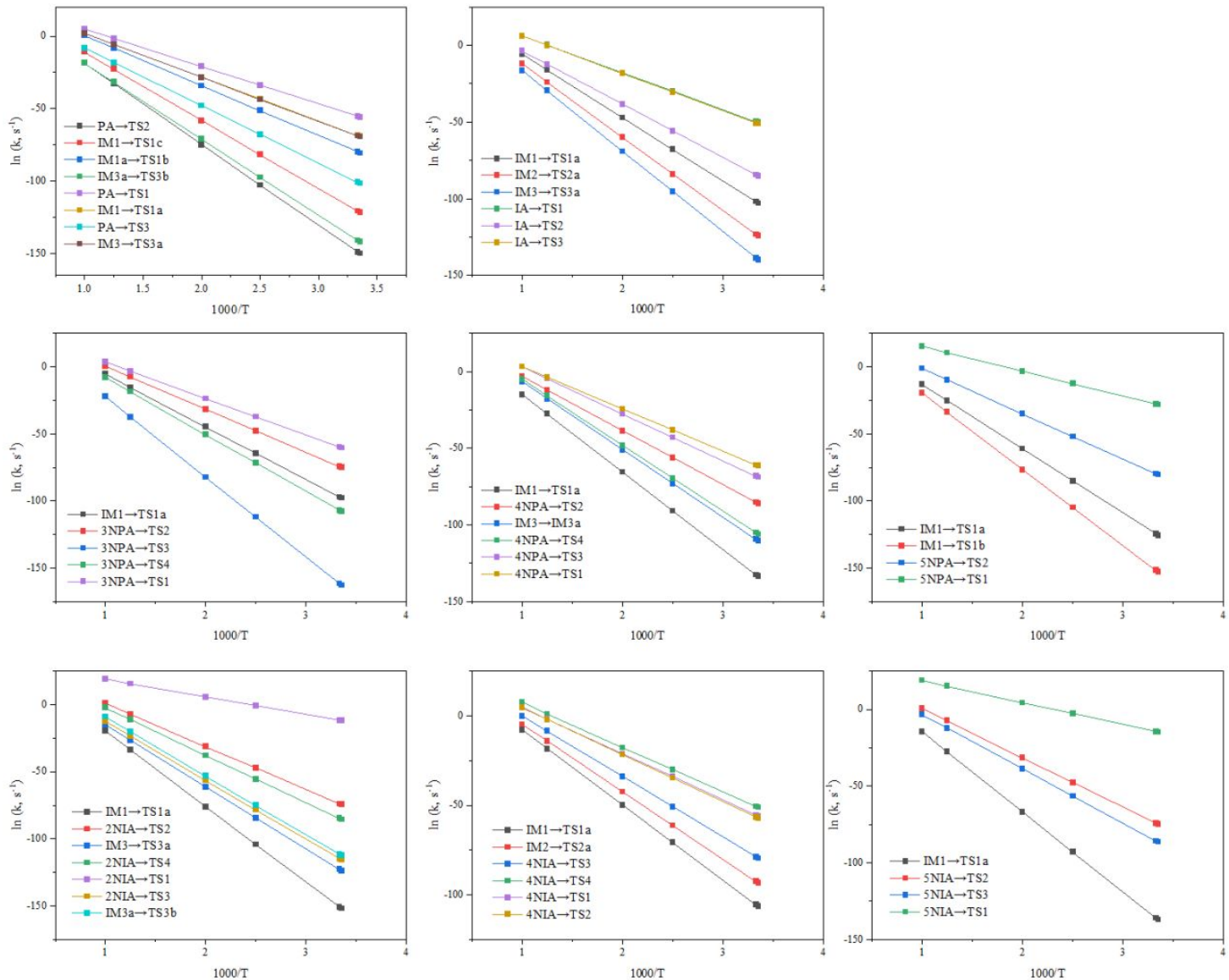

Figure S12. The logarithmic dependences of rate constants $(\ln k)$ on temperatures $(1000 / \mathrm{T})$ for initial decomposition channels of all channels of PA, IA and nitro-derivatives. 